\title{
Variants of Concern responsible for SARS-CoV-2 vaccine breakthrough infections from India
}

Urvashi B Singh", ${ }^{, *}$, Mercy Rophina ${ }^{2,3, *}$, Rama Chaudhry ${ }^{1}$, Vigneshwar Senthivel ${ }^{2,3}$, Kiran Bala ${ }^{1}$, Rahul C. Bhoyar $^{2}$, Bani Jolly ${ }^{2,3}$, Nayer Jamshed ${ }^{5}$, Mohamed Imran ${ }^{2,3}$,Ritu Gupta ${ }^{4}$, Praveen Aggarwal ${ }^{5}$, Mohit Kumar Divakar $^{2,3}$, Subrata Sinha ${ }^{6}$, Arvinden VR $^{2,3}$, Anjali Bajaj ${ }^{2,3}$, Afra Shamnath ${ }^{2}$, Abhinav Jain ${ }^{2,3}$, COVID CBNAAT CORE GROUP $\$$, Vinod Scaria ${ }^{2,3^{\wedge}}$, Sridhar Sivasubbu ${ }^{2,3^{\wedge}}$, Randeep Guleria ${ }^{7}$

${ }^{1}$ Department of Microbiology, All India Institute of Medical Sciences, New Delhi

${ }^{2}$ CSIR Institute of Genomics and Integrative Biology (CSIR-IGIB), Mathura Road, New Delhi, India

${ }^{3}$ Academy for Scientific and Innovative Research, Human Resource Development Centre Campus, Ghaziabad, Uttar Pradesh, India

${ }^{4}$ Department of Laboratory Oncology, All India Institute of Medical Sciences, New Delhi

${ }^{5}$ Department of Emergency Medicine, All India Institute of Medical Sciences, New Delhi

${ }^{6}$ Department of Biochemistry, All India Institute of Medical Sciences, New Delhi

${ }^{7}$ Department of Pulmonary, Critical Care \& Sleep Medicine, All India Institute of Medical Sciences, New Delhi

*Equally contributed

$\$$ The members of the COVID CBNAAT CORE GROUP are listed in the Supplementary Appendix

$\wedge$ Senior Authors, equally contributed

\#Address for correspondence:

Dr. Urvashi B. Singh MD PhD FAMS

Professor

Department of Microbiology

All India Institute of Medical Sciences,New Delhi-110029

Mobile No. +919811120203

Fax: +91 1126588663

Email : drurvashi@gmail.com 


\begin{abstract}
Emerging reports of SARS-CoV-2 breakthrough infections entail methodical genomic surveillance for determining efficacy of vaccines. This study elaborates genomic analysis of isolates from breakthrough infections following vaccination with AZD1222/Covishield and BBV152/Covaxin.Variants of concern B.1.617.2 and B.1.1.7 responsible for cases surge in April-May 2021 in Delhi, were the predominant lineages among breakthrough infections.
\end{abstract}




\section{INTRODUCTION}

Vaccines have been one of the most powerful weapons against infectious diseases. The emergence of COVID-19 as a global pandemic has seen accelerated development as well as regulatory approvals for a variety of vaccines across the world. Immunity acquired from natural COVID-19 infection or vaccines is not absolute and reinfections post natural COVID-19 infections as well as vaccine breakthrough infections are not uncommon (1),(2),(3). The recent evidence from the United States suggests vaccine breakthrough infections to be approximately $0.01 \%$ in fully vaccinated populations(4). One of the initial surveillance reports from a chronic care medical facility in India reported out of a total of 113 employees, 19 experienced vaccine breakthrough infections(5).

Genomic surveillance of vaccine breakthrough infections can provide useful insights into the genetic variants of SARS-CoV-2 that caused the infection (2),(3). In this study, we describe the genomic analysis of a cohort of COVID-19 vaccine breakthrough infections in a tertiary health care centre in Delhi, India. The samples included in the study coincided with a COVID-19 cases surge in the state during the months of April and May 2021. 


\section{RESULTS AND DISCUSSIONS}

Our analysis included 63 cases of vaccine breakthrough infections for which the dates of vaccines could be ascertained, of which 36 patients received two doses, while 27 had received one dose of vaccine. Ten patients received AZD1222/Covishield while 53 received BBV152/Covaxin. The timeline for vaccination and infection in the context of the surge in cases in Delhi is summarised in Figure 1A. The patients had a mean age of 37 (21-92), in which 41 were males and 22 were females. None of the patients had any comorbidities which could act as a predisposing factor for breakthrough infections.

The mean depth of coverage of the genome sequences for the 63 viral isolates was 3953X (108.7X17435.5X) and it correlated with the viral load as reflected by the Ct values. The patient details and genomic data are summarised in Supplementary Table S1. A phylogenetic tree was constructed following a previously published protocol(6) using B.1.617.1 and B.1.617.2 genomes from India submitted to GISAID. Genome sequences of 16 of the 63 viral isolates that had $<=2000$ ambiguous bases were included in the phylogenetic analysis. The clustering of the 16 samples on the phylogenetic tree is suggestive of multiple independent sources of infection for the patients analysed. The phylogenetic context of the samples sequenced is summarised in Figure 1B.

Lineages were systematically assigned to the genomes analysed in this study using pangolin 2.4 (pangoLEARN version 2021-04-28)(7). SARS-CoV-2 lineages could be assigned for a total of 36 (57.1\%) samples, $19(52.8 \%)$ in patients who completed both doses and $17(47.2 \%)$ in patients who completed only a single dose. B.1.617.2 was found to be the predominant lineage with 23 samples 
$(63.9 \%)$ out of which 12 were in fully vaccinated and 11 in partially vaccinated groups. $4(11.1 \%)$ and $1(2.8 \%)$ samples were assigned the lineages B.1.617.1 and B.1.1.7 respectively. The B.1.617.2 lineage was first described in India and associated with increased transmissibility as well as immune escape and has grown to become one of the predominant lineages in India.

Additional samples covering the respective period (March 28, 2021 - April 30, 2021) from Delhi with no prior vaccination information, deposited in GISAID were used as the control dataset. As lineage B.1.617.2 was also prevalent in this group, any significant differences in lineages among fully and partially vaccinated samples were analysed. The difference was not found to be significant in both double dose vaccinated and single dose vaccinated groups (Vaccine-Double dose $: \chi^{2}$ corrected $=$ 0.0112 , pval $=0.9159 ;$ Vaccine-Single dose $: \chi^{2}$ corrected $=3.2441$, pval $\left.=0.0717\right)$. The proportions of lineages are summarised in Figure 1C. In addition, differences in the prevalence of lineages based on the type of vaccines were also checked. There was no significant difference observed $\left(\chi^{2}\right.$ corrected $=$ 0.0586, pval $=0.8087$.

Of the breakthrough infection cases analysed, 10 patients ( 8 with double doses of vaccine and 2 with single vaccine dose) additionally had total Immunoglobulin $\mathrm{G}$ (IgG) antibodies assessed by Chemiluminescent Immunoassay (Siemens), of which 6 patients had IgG antibodies a month before the infection, while 4 had antibodies after the disease episode.

Viral load at the time of diagnosis was high in all the patients irrespective of vaccination status or type of vaccine received. The initial course of disease with high-grade non-remitting fever lasted for five to seven days in the vaccinated group, similar to the clinical presentation in unvaccinated patients. During 
the subsequent course of illness, neither disease worsening (stable biomarkers) nor mortality was reported in the present group, confirming the previous observations.

The patients included health care workers (24, 13 of which were from the same hospital) and close analysis of the genomic sequences suggest that the samples clustered separately with origins closely clustering with lineages from different states, suggesting the disease transmission happened most likely from different and independent sources.

Reinfections and vaccine breakthrough infections are rare occurrences and genomic sequencing of vaccine breakthrough infections can provide useful insights. In the present group of vaccine breakthrough infections investigated using genome sequencing, closely overlapping and mirroring the COVID-19 cases in the state of Delhi, the variants of concern B.1.617.2 and B.1.1.7 comprised the majority, but the proportions were not significantly different in comparison with the population prevalence of the variants during this period with high community transmission. Breakthrough infections reported to CDC have primarily been attributed to variants of concern and the proportion was similar to the prevalence throughout United States, again during a period of high transmission.(4) While a number of vaccine breakthrough infections have been reported previously, it has been largely associated with non-severe symptoms. Mortality due to COVID-19 was ascribed to $2 \%$ cases (primarily older population, average age-82 years)(4). Of the 63 cases of vaccine breakthrough infections in the current study, including 36 who received full doses, there are no reports of mortality even though almost all cases presented with high-grade unremitting fever for 5-7 days. While total antibody levels for a subset of patients were high, they became infected nevertheless and presented to 
the emergency just like other patients, putting in doubt the protection offered and or clinical relevance of total IgG as a surrogate of COVID-19 immunity.

The present report is unique in many aspects. This is one of the first reports of symptomatic vaccine breakthrough infections, and breakthrough infections in a tertiary care setting apart from being the earliest reports of breakthrough infections with BBV152/Covaxin and B.1.617.2 variant of SARSCoV-2. This report brings forth the role of highly transmissible variants of concern in causing breakthrough infections in the vaccinated population. $(3,12)$ We hope this early report of genomic analysis of breakthrough infections could provide guidance to further systematic cohort analysis on the efficacy of vaccines with respect to genetic variants of SARS-CoV-2. 


\section{MATERIALS AND METHODS}

\section{Study approval}

Samples were collected as part of routine testing for patients reporting to the Emergency Department at the All India Institute of Medical Sciences, with symptoms of high grade fever, shortness of breath and headache. The samples were subjected to whole-genome sequencing following the IEC approvals (IEC-679/03.07.2020,RP-32/2020).

All individuals who completed the schedule of vaccine and tested positive for SARS-CoV-2 on quantitative real-time polymerase chain reaction (qRT-PCR) test (Genexpert SARS-CoV-2, Cepheid, Sunnyvale, USA and TRUNAT Beta-CoV, Molbio India), were included in the analysis. The cohort was subdivided into two groups depending on whether they received only one dose or both doses as per the vaccination schedule. Additional metadata including the dates of vaccination, type of vaccine received, dates of testing positive, clinical information including symptoms and history of forward transmission as well as occupation were elicited. All samples which did not satisfy the criteria or patients who could not be reached were duly excluded.

The daily COVID-19 cases in Delhi were retrieved from an open resource(8). Genomic datasets covering the period were also retrieved from Global Initiative on Sharing All Influenza Data (GISAID)(9) (Supplementary Data 1) for comparison on variant profiles.

Whole-genome sequencing was performed on the SARS-CoV-2 positive RNA samples, using the COVIDSeq Test (Illumina Inc.,) assay in NovaSeq 6000 platform adopting a paired-end sequencing approach with $50 * 2$ read length(10) and analysed as per published protocol(11). 


\section{AUTHOR CONTRIBUTIONS}

UBS formulated the study with VSc and SS. UBS and VSc planned the work, and wrote the manuscript with MR. UBS, RC, KB, SS, RiG contributed to diagnostics, laboratory management, CCCG conducted diagnostic tests, NJ, PA and RG contributed to clinical data collection and clinical care. VSe, RB, MKD, MI and AB contributed to the NGS data generation under the guidance of SS. $\mathrm{MR}, \mathrm{BJ}$ and $\mathrm{AV}, \mathrm{AS}$ and $\mathrm{AJ}$ contributed to the data analysis.

UBS conceived the project and followed up the clinical data and MR was involved in genomic data analysis.

\section{ACKNOWLEDGEMENTS}

Authors acknowledge funding from the Council of Scientific and Industrial Research (CSIR India) through grants CODEST and MLP2005. BJ, MKD, AB, AJ acknowledge fellowship from CSIR India. SS acknowledges the J C Bose Fellowship of the Dept of Science and Technology, India. See Supplemental appendix for COVID CBNAAT CORE GROUP details.

\section{CONFLICTS OF INTEREST}

Authors declare no conflicts of interest.

The funding agencies had no role in the analysis of data, preparation of manuscript or decision to publish.

\section{DATA AVAILABILITY}

The sequence data is available in GISAID with IDs EPI_ISL_2426235- EPI_ISL_2426245 


\section{FIGURE}

(A)

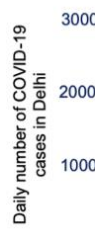

(20)

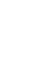

.
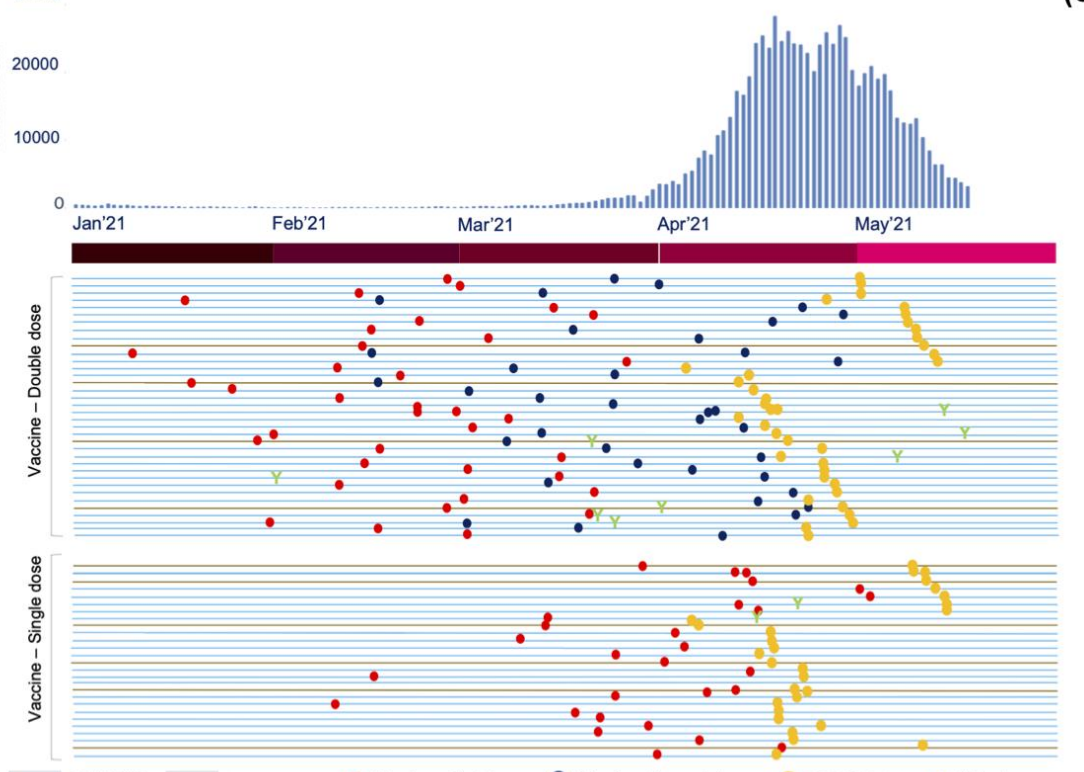

$\square$ COVAXIN $\square$ COVISHIELD Vaccine-First dose Vaccine-Second dose RT-PCR Test Y Ab Tests

(B)

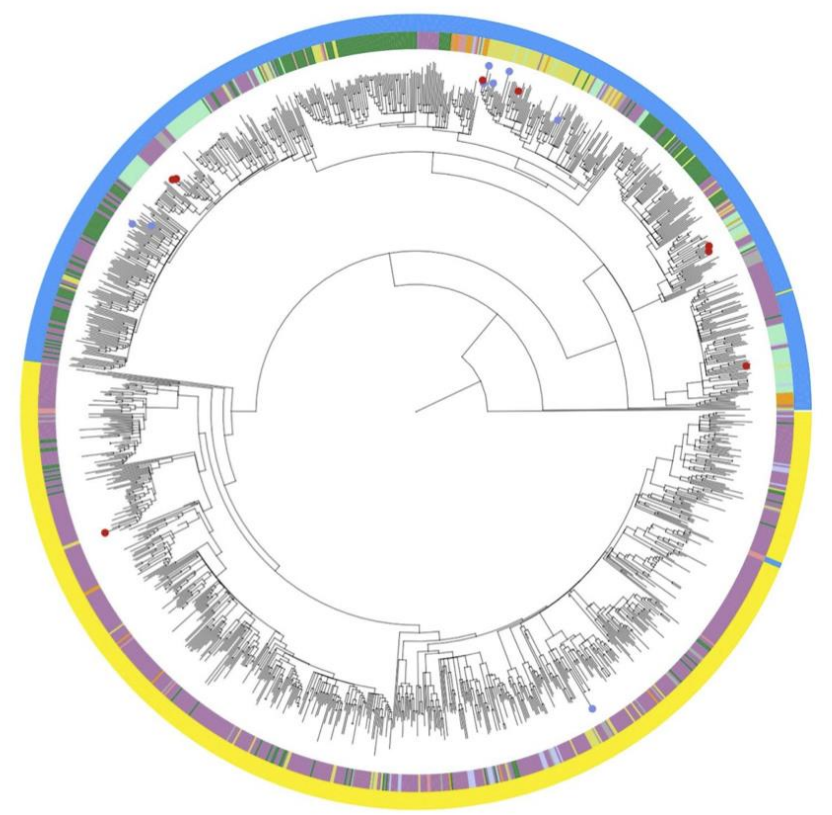

Double Dose

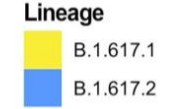

Location

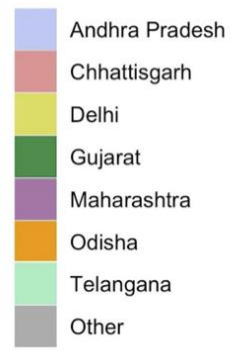

Figure 1. (A) Clinical timeline of the samples used in the study in context with the daily surge of cases in Delhi (B) Phylogenetic context of the SARS-CoV-2 isolates analysed in the study (C) Proportions of lineages in categories of samples with double, single vaccine doses and in controls from a period of March 28, 2021 to April 30, 2021. 


\section{SUPPLEMENTARY DATASETS}

Supplementary Data 1 : GISAID acknowledgement table for the samples used in the study

Summary of samples from public sources used in the study

Supplementary Table S1 : Summary of vaccine breakthrough infection samples used in the study

Breakthrough infection samples summary

Supplementary Appendix : The list of the COVID CBNAAT CORE GROUP members COVID CBNAAT CORE GROUP members 


\section{REFERENCES}

1. Mukherjee A, Anand T, Agarwal Anup et al. SARS-CoV-2 re-infection: development of an epidemiological definition from India [Internet]. Epidemiol. Infect. 2021;149.

doi:10.1017/S0950268821000662

2. Philomina BJ, Jolly B, John N et al Genomic survey of SARS-CoV-2 vaccine breakthrough infections in healthcare workers from Kerala, India [Internet]. J. Infect. [published online ahead of print: May 25, 2021]; doi:10.1016/j.jinf.2021.05.018

3. Hacisuleyman E, Hale C, Saito Y et al. Vaccine Breakthrough Infections with SARS-CoV-2 Variants [Internet]. N. Engl. J. Med. [published online ahead of print: April 21, 2021]; doi:10.1056/NEJMoa2105000

4. Birhane M, Bressler S, Chang G et al. COVID-19 Vaccine Breakthrough Infections Reported to CDC — United States, January 1-April 30, 2021 [Internet]. MMWR Morb. Mortal. Wkly. Rep. 2021;70. doi:10.15585/mmwr.mm7021e3

5. Tyagi K, Ghosh A, Nair D et al. Breakthrough COVID19 infections after vaccinations in healthcare and other workers in a chronic care medical facility in New Delhi, India. Diabetes \& Metabolic Syndrome: Clinical Research \& Reviews 2021;15(3):1007-1008.

6. Jolly B, Scaria V. Computational analysis and phylogenetic clustering of SARS-CoV-2 genomes [Internet]. Bio Protoc. 2021;11(8). doi:10.21769/bioprotoc.3999

7. Rambaut A, Holmes EC, O’Toole et al. A dynamic nomenclature proposal for SARS-CoV-2 lineages to assist genomic epidemiology. Nat Microbiol 2020;5(11):1403-1407. 
8. Coronavirus in India: Latest Map and Case Count [Internet]https://www.covid19india.org. cited May 27, 2021

9. Shu Y, McCauley J. GISAID: Global initiative on sharing all influenza data - from vision to reality [Internet]. Euro Surveill. 2017;22(13). doi:10.2807/1560-7917.ES.2017.22.13.30494

10. Bhoyar RC, Jain A, Sehgal P et al. High throughput detection and genetic epidemiology of SARSCoV-2 using COVIDSeq next-generation sequencing. PLoS One 2021;16(2):e0247115.

11. Poojary M, Shantaraman A, Jolly B, Scaria V. Computational Protocol for Assembly and Analysis of SARS-nCoV-2 Genomes [Internet]. rr2020;4.http://www.companyofscientists.com/index.php/rr/article/view/165. cited May 28, 2021

12. Jolly B, Rophina M, Shamnath A et al. Genetic epidemiology of variants associated with immune escape from global SARS-CoV-2 genomes. bioRxiv preprint 2021; doi: https://doi.org/10.1101/2020.12.24.424332; 